\title{
Comparison of the efficacy of different progesterone regimens in blastocyst frozen-thawed embryo transfer cycles
}

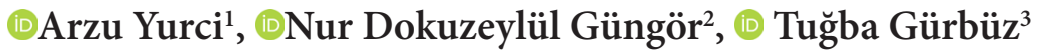 \\ ${ }^{1}$ Memorial Hospital, IVF Clinic, Kayseri, Turkey \\ ${ }^{2}$ Bahçeşehir University Göztepe Medical Park Hospital Complex, Obstetrics and IVF Clinic, İstanbul, Turkey \\ ${ }^{3}$ Medistate Hospital, Gynecology and Obstetric Clinic, İstanbul, Turkey
}

Cite this article as: Yurci A, Dokuzeylül Güngör N, Gürbüz T. Comparison of the efficacy of different progesterone regimens in blastocyst frozen-thawed embryo transfer cycles. J Health Sci Med 2021; 4(2): 137-141.

\begin{abstract}
Aim: The progesterone's optimal route for luteal phase support (LPS) in frozen-thawed embryo transfer (FET) cycles is controversial. This study investigates the effect of three treatment regimens of progesterone: vaginal, oral, and subcutaneous form for LPS in FET cycles.

Material and Method: Blastocyst cryopreserved FET cycles utilizing vaginal, subcutaneous, or oral forms of progesterone between December 2018 and May 2020 were included. The main outcome was to compare clinical pregnancy rates (CPR), ongoing pregnancy rates (OPR), and live birth rates (LBR) in different progesterone groups.

Results: A total of 434 cycles were included, of which 200 utilized vaginal, 124 utilized subcutaneous, and 110 utilized oral forms. Demographic and cycle characteristics were similar between all three groups. Compared to cycles utilizing vaginal, subcutaneous, and oral forms, CPR, OPR, and LBR differ significantly between the three groups ( $p$-value $=0.000$ ). Miscarriage was calculated according to the number of days after embryo transfer, and it was shown that the subcutaneous group had the lowest rate of miscarriage with 11 cases (8.8\%). The highest OPR was associated with the subcutaneous group with 67 (54\%), followed by the vaginal group with $92(46 \%)$. The highest LBR was in the subcutaneous group, with $59(47.6 \%)$ cases. The oral group was significantly less successful $29(26.4 \%)$ than the subcutaneous and vaginal groups.

Conclusion: Our study results showed that subcutaneous and vaginal progesterone performed better than oral progesterone for LPS in patients undergoing FET. All three forms of progesterone administration were safe and well-tolerated.
\end{abstract}

Keywords: Frozen-thawed embryo transfer, luteal phase support, progesterone, in vitro fertilization

\section{INTRODUCTION}

Frozen-thawed embryo transfer (FET) is one of the most important advances in assisted reproductive techniques (ART) that has many benefits for patients (1). Using this method and transferring fewer embryos reduces the risk of multiple pregnancies and increases the patient's chances of getting pregnant using multiple embryos without additional stimulation with stimulated cycles (2). Also, by FET in patients at risk for ovarian hyperstimulation syndrome, this syndrome's risk is greatly reduced (3).

Progesterone is usually used as luteal support for frozen embryo transfer due to increasing implantation and pregnancy rate $(1,4,5)$. The FET process's success depends on the progesterone support secreted by the corpus luteum, known as luteal phase support (LPS) (6). This support increases the rate of pregnancy after ART procedures (7). Today, LPS in ovulation induction and FET cycles by progesterone products have attracted a lot of attention, which increases the rate of pregnancy and reduces the chance of miscarriage (8-10).

Various studies have been performed on the type of progesterone for LPS (1-9). Different types of progesterone are used for this purpose, including

1) Oral micronized progesterone (300-600 mg per day): One of this method's advantages is easy administration, but it is metabolized quickly by the liver. Its absorption rate is different in different people. Dydrogesterone, as the optical isomer of progesterone, is similar in formulation and structure to natural progesterone, and no specific side effects have been reported $(10,11)$. 
2) Subcutaneous progesterone injection (25 $\mathrm{mg}$ twice daily): Requires daily injection and may cause a local reaction at the injection site. Its advantages are comfortable, easy to use, and efficient (12).

3) Vaginal progesterone: in the form of a gel (Crinone $8 \%$ of an applicator $90 \mathrm{mg}$ per day) of the advantages of the vaginal method is the easy administration, but the relatively expensive price and the rare cases of vaginal irritation, inflammation, itching, leakage, and bleeding are its disadvantages (20).

Today, vaginal progesterone is more widely used in medical centers due to the high concentration of progesterone and its direct effect on the endometrium (20). The preferred regimen of LPS in FET cycles is unclear. Therefore, this study investigates the effect of three treatment regimens for LPS in FET cycles: vaginal, subcutaneous, and oral progesterone.

\section{MATERIAL AND METHOD}

This retrospective cohort study was approved by the Ethics Board of Erciyes University (number 2020/341, date 24.06.2020). The study was performed on 434 patients referred to the Kayseri Memorial In Vitro Fertilisation (IVF) Center from December 2018 to May 2020. All procedures performed in studies involving human participants were in accordance with the ethical standards of the institutional and/or national research committee and with the 1964 Helsinki declaration and its later amendments or comparable ethical standards. All patients provided their informed consent before enrolling.

All woman undergoing FET cycle at this time period were given vaginal, subcutaneous or oral progesterone for LPS according to patient preference or physician preference.

Patients with unknown infertility problems and women using birth control methods were excluded from the study. The mean age of participants was 33.5 , with a minimum age of 20 and a maximum age of 39 . Older subjects were excluded from the study.

Participants were divided into three groups: receiving progesterone vaginal, oral, and subcutaneous. There were 200 people in the vaginal group, 124 in the subcutaneous group, and 110 in the oral group. Participants in the first group received $90 \mathrm{mg}$ of vaginal progesterone twice daily (Crinone gel, Merck, United Kingdom). The second group received $25 \mathrm{mg}$ of subcutaneous progestogen twice a day (Prolutex, IBSA, Switzerland). The third group received $10 \mathrm{mg}$ of oral dydrogesterone three times a day (Duphaston, Abbott Healthcare Products, Netherlands).
The routine IVF protocol utilized at our center was started with an initial examination performed on the first three days of menstruation. Briefly, patients' endometrial development was serially monitored with ultrasonography by evaluating endometrial thickness, pattern, and the presence/ absence of active or residual follicles. Also, estradiol and progesterone's blood levels were determined and recorded, again with routine serial measurements. In preparation for IVF, patients were started on estradiol valerate $2 \mathrm{mg}$ tablet (Estrofem tablet, three times daily), roxithromycin $300 \mathrm{mg}$ tablet (Rulid tablet, once daily), acetylsalicylic acid $100 \mathrm{mg}$ tablet (Coraspin tablet, once daily), and folic acid $5 \mathrm{mg}$ tablet (Folbiol tablet, once daily) on the second day of menstruation. Patients continued to receive these treatments until the 12th day of menstruation. Progesterone initiation was begun after detecting 8-mm endometrium thickness and observing the characteristic "triple line" pattern. Progesterone was given through oral, vaginal, and subcutaneous applications to induce endometrial secretion to transfer the thawed embryo. As a rule of thumb, normal transfers were planned on the 18th or 19th days of the menstrual cycle, corresponding to 5 days of embryo development. Embryo transfers were performed under ultrasound guidance using a soft tip catheter (Cook Medical, USA) on the sixth day of progesterone administration. One or two blastocyst embryo transfer was performed at the stage of top quality or good quality embryos according to Gardner and Schoolcraft blastocyst grading system. Estradiol and progesterone supplementation were continued until the day of the pregnancy test, carried out 10 days after the embryo transfer. If the test was positive, estradiol and progesterone was maintained due to 10th gestational week.

Main outcome was to detect clinical pregnancy rate, ongoing pregnancy rate and live birth rate according to different progesterone regimens. Clinical pregnancy was defined as the confirmation of an intrauterine gestational sac at 6-7 weeks of pregnancy. Ongoing pregnancy was defined as a pregnancy proceeding beyond 20th gestational week and live birth was defined as the delivery of a living newborn after 24th gestational week. Miscarriage was defined as pregnancy loss after detection of a fetal heartbeat during TvUSG exam and before 20th gestational week.

\section{Statistics}

Chi-square test was used to examine the significant difference between each of the qualitative variables in the three groups. If the number of data in at least one cell was less than the other cells in the agreement table, Fisher's exact test was used and ${ }^{*}$ denotes these variables. For quantitative variables, after examining their abnormality using the Kolmogorov-Smirnov test, the non-parametric equivalent of a one-way ANOVA test, the Kruskal-Wallis test was used. Statistical Package for Social Sciences (SPSS) version 26.0 (SPSS Inc., Chicago, IL, USA) was used to perform analysis. 


\section{RESULTS}

There were 443 patients, and the patients were divided into three groups according to the progesterone administration route as vaginal, subcutaneous, and oral. The descriptive statistics of the participants are given in Table 1. The frequency of descriptive statics of patients within groups is also shown in Table 2. There was no significant difference between the descriptive statistics of the participants in the three different groups.

\begin{tabular}{|lcc|}
\hline \multicolumn{3}{|l|}{ Table 1. The descriptive statics of patients } \\
\hline Variable & Mean (Sd) & Min-Max \\
\hline Age & $33.5(4.7)$ & $20-39$ \\
Cycles day in transfer & $16.9(1.4)$ & $16-19$ \\
Embryo day & $5(0)$ & $5-5$ \\
Endometrium thickness & $10.45(1.33)$ & $8-16$ \\
\hline
\end{tabular}

\begin{tabular}{|c|c|c|c|}
\hline \multirow{2}{*}{ Variable } & \multicolumn{3}{|c|}{ Frequency (\%) within groups } \\
\hline & Vaginal & Subcutaneous & Oral \\
\hline Age & $34.3(4.9)$ & $35.6(3.9)$ & $29.5(2.5)$ \\
\hline Cycles day in transfer & $17.4(0.9)$ & $15.29(1.07)$ & $17.9(1.2)$ \\
\hline Embryo day & $5(0)$ & $5(0)$ & $5(0)$ \\
\hline Endometrium thickness & $10.37(1.29)$ & $10.48(1.28)$ & $10.57(1.4)$ \\
\hline
\end{tabular}

As shown in Table 1, the participants' minimum age was 20 , and the maximum age was 39 (mean \pm SD: $33.5 \pm 4.7$ ). Also, the cycle days spent during the experiment for participants were a minimum of 16 days and a maximum of 19 days (mean \pm SD: $16.9 \pm 1.4$ ). The day of receiving the embryo was the fifth day for all participants (Mean \pm SD: $5 \pm 0$ ). The endometrium thickness was a minimum of $8 \mathrm{~mm}$ and a maximum of $16 \mathrm{~mm}$ in the participants (mean \pm SD: $10.45 \pm 1.33$ ).

The descriptive statistics of the pregnancy variables are presented in Table 3. As shown in Table 3, in the participants' pregnancy beta test, 166 (38.2\%) subjects were negative, and $268(61.8 \%)$ were positive. The next examination was to test for the gestational sac formation. The examination result was negative for 167 (38.5\%) subjects and positive for 267 (61.5\%). The fetal heart rate was then examined, and results were negative for 173 (39.9\%) subjects and positive for 261 (60.1\%). The next field is related to miscarriage, which is divided according to the days after embryo transfer. The results show that $355(81.8 \%)$ subjects did not experience a miscarriage. Also, $1(0.2 \%)$ subject on the sixth day, $31(7.1 \%)$ on the seventh day, $31(7.1 \%)$ on the eighth day, and $16(3.7 \%)$ on the ninth day experienced a miscarriage. The next field in the table shows that the child was born at the right time. This result was negative for $282(65 \%)$ participants and positive for $152(35 \%)$. The frequency and p-value of pregnancy variables within groups are shown in Table 4.

\begin{tabular}{|lccc|}
\hline \multicolumn{4}{|l}{ Table 3. The descriptive statistics pregnancy variables } \\
\hline Variable & Value & Frequency & Percent (\%) \\
\hline \multirow{2}{*}{ Beta pregnancy test } & 0 & 166 & 38.2 \\
& 1 & 268 & 61.8 \\
Gestational sac & 0 & 167 & 38.5 \\
Heartbeat & 1 & 267 & 61.5 \\
& 0 & 173 & 39.9 \\
& 1 & 261 & 60.1 \\
Miscarriage & 0 & 355 & 81.8 \\
& 6 & 1 & 0.2 \\
& 7 & 31 & 7.1 \\
Live birth rate & 8 & 31 & 7.1 \\
Ongoing pregnancy & 0 & 16 & 3.7 \\
& 1 & 282 & 65 \\
& 0 & 152 & 35 \\
\hline
\end{tabular}

\begin{tabular}{|c|c|c|c|c|c|}
\hline \multirow[b]{2}{*}{ Variable } & \multirow[b]{2}{*}{ Value } & \multirow[b]{2}{*}{ Vaginal } & \multicolumn{2}{|c|}{$\begin{array}{l}\text { Frequency (\%) } \\
\text { within groups }\end{array}$} & \multirow[b]{2}{*}{$\begin{array}{c}p \\
\text { value }\end{array}$} \\
\hline & & & Subcutaneous & Oral & \\
\hline \multirow{2}{*}{$\begin{array}{l}\text { Beta } \\
\text { pregnancy } \\
\text { test }\end{array}$} & 0 & $56(28)$ & $45(36.3)$ & $65(59.1)$ & \multirow[b]{2}{*}{0.000} \\
\hline & 1 & $144(72)$ & $79(63.7)$ & 45 (40.9) & \\
\hline \multirow{2}{*}{$\begin{array}{l}\text { Gestational } \\
\text { sac }\end{array}$} & 0 & $56(28)$ & $46(37.1)$ & $65(59.1)$ & \multirow{2}{*}{0.000} \\
\hline & 1 & $144(72)$ & $78(62.9)$ & 45 (40.9) & \\
\hline \multirow{2}{*}{ Heartbeat } & 0 & $60(30)$ & 48 (38.7) & $65(59.1)$ & \multirow{2}{*}{0.000} \\
\hline & 1 & $140(70)$ & $76(61.3)$ & 45 (40.9) & \\
\hline \multirow{5}{*}{ Miscarriage $^{\star}$} & 0 & $148(74)$ & $113(91.1)$ & $94(85.5)$ & \multirow{5}{*}{0.001} \\
\hline & 6 & 0 & $1(0.8)$ & 0 & \\
\hline & 7 & $21(10.5)$ & $5(4)$ & $5(4.5)$ & \\
\hline & 8 & $18(9)$ & $3(2.4)$ & $10(9.1)$ & \\
\hline & 9 & $13(6.5)$ & $2(1.6)$ & $1(0.9)$ & \\
\hline Total & & $52(26)$ & $11(8.8)$ & $16(14.5)$ & \\
\hline \multirow{2}{*}{$\begin{array}{l}\text { Live birth } \\
\text { rate }^{*}\end{array}$} & 0 & $130(65)$ & $65(52.4)$ & $87(79.1)$ & \multirow{2}{*}{0.000} \\
\hline & 1 & $70(35)$ & $59(47.6)$ & $23(20.9)$ & \\
\hline Ongoing & 0 & $108(54)$ & $57(46)$ & $81(73.6)$ & \multirow{2}{*}{0.000} \\
\hline pregnancy & 1 & $92(46)$ & $67(54)$ & $29(26.4)$ & \\
\hline
\end{tabular}

The last field indicated Ongoing pregnancy, which means a successful pregnancy. The participants' results showed that this variable was negative for 246 (56.7\%) subjects and positive for 188 (43.3\%).

As Table 4 shows, Beta pregnancy test results differ significantly between the three groups ( $\mathrm{p}$-value $=0.000$ ). These results showed that the highest positive Beta pregnancy test was related to the vaginal group, with 144 (72\%) cases. The subcutaneous group with 79 (63.7\%) positive cases and the oral group, with 45 (40.09\%) positive cases were also observed. The results of the gestational sac test also showed similar results. 
The fetal heartbeat test showed that the highest positive rates were observed in the vaginal group with 140 (70\%), followed by the subcutaneous group with 76 (61.3\%) and the oral group with $45(40.9 \%)$. These results were significantly different in the three groups ( $\mathrm{p}$-value $=0.000$ ).

Miscarriage was calculated according to the number of days after embryo transfer, and it was shown that the subcutaneous group with $11(8.8 \%)$ had the lowest rate of miscarriage, followed by the oral group with $16(14.5 \%)$ and the vaginal group with $52(26 \%)$. Also, the seventh and eighth days with 31 cases recorded the highest miscarriage in a day.

Comparing the live birth rate showed a significant difference between the three groups ( $\mathrm{p}$-value $=0.000$ ). The highest rates of live birth were associated with the subcutaneous group with 59 (47.6\%), followed by the vaginal group with $70(35 \%)$ and the oral group with 29 $(26.4 \%)$.

Finally, the results of successful pregnancies in participants also showed a significant difference between the three groups $(p<0.001)$ : the highest successful pregnancy rate was associated with the subcutaneous group with 67 (54\%), followed by the vaginal group with $92(46 \%)$ and the oral group with $29(26.4 \%)$ cases. These results showed that the oral group was significantly less successful than the subcutaneous and vaginal groups.

\section{DISCUSSION}

There has been increasing trend in the use of frozen embryo transfer cycles in recent years. Although various protols have been used to prepare the endometrium during FET cycles, the ideal protocol for endometrial preparation and LPS in FET cycles is controversial yet. Endometrial and embryo coherence in frozen-thawed embryo transfer (FET) cycles is influenced by various factors, including the duration and type of progesterone treatment before embryo transfer (5).

Progesterone preparations can be administered by vaginal, subcutaneous, oral or intramuscular, routes. Vaginal progesterone preparations are considered as more patient-friendly; however, they may cause vaginal irritation, discomfort, and may not be preferred by some women owing to cultural reasons or medical conditions like vaginismus (13). Subcutanoeous progesterone preparations are the water-soluble forms of progesterone which is most recently developed. It was shown that absorbtion is faster and reaches peak serum concentration quicker than intramuscular progesterone (14).

Except dydrogesterone, other oral progesterone preparations are not suitable for LPS bacause of low bioavailabilities after hepatic first passage effect (15).
Our study examined three different progesterone preparations for LPS undergoing FET cycles. We found that the pregnancy outcomes including CPR, OPR and LBR were all significantly lower in the oral progesterone group than the other groups, while the vaginal and subcutaneous groups had comparable pregnancy outcomes in this regard. These results suggest that vaginal and subcutaneous progesterone are acceptable supplementations for LPS, but oral supplementation may not provide adequate LPS. This result of our study is consistent with a randomised controlled trial in the literature which compared oral dydrogesterone, vaginal progesterone, human corionic gonadotropin plus oral dydrogesterone and gonadotropin releasing hormone analogue plus oral dydrogesterone in FET cycles (16). Only oral dydrogesterone group was found to have lower pregnancy rates than the others. But there is also conflicting results in the literature concerning the efficacy of oral dydrogesterone for LPS in FET cycles. Guo et al. (17) investigated subcutaneous dydrogesterone and oral progesterone for LPS in FET cycles and concluded that oral dydrogesterone produces better results for LPS the FET cycles. Rashidi et al. (18) compared pregnancy outcomes of oral dydrogesterone, intramuscular progesterone and vaginal progesterone suppository use in FET cycles and found CPR $36.6 \%, 38.3 \%$ and $28.3 \%$ respectively and LBR $30 \%, 28.3 \%$ and $26.6 \%$ respectively which were similar between the groups. This result might be associated with a relatively high dosage of oral progesterone that $40 \mathrm{mg}$ daily oral dydrogesterone was used in this trial.

In a recent randomised trial, LPS for FET cycles was performed by vaginal route, intramuscular route and their combination (19). Miscarriage rate was higher in the vaginal progesterone only group that resulted in significantly lower ongoing pregnancy rates than intramusculer route and the combination. This result might be considered as compatible with our study's miscarriage results that we also found higher miscarriage results in the vaginal and oral progesterone groups than the subcutaneous group.

One of the limitations of this study and studies in this area is the retrospective nature of the study. More detailed randomized clinical trials are needed to examine the efficacy of progesterone forms/combinations more accurately. In the same way with our study, pregnancy outcomes with different progesterone forms for LPS were measured only in quality embryos in the literature, while the results may be different in lower quality embryos. Real-world outcomes may be different from the selected study groups.

According to current literature, the available evidence on the optimal progesterone preparation in FET cycles is far from conclusive, and randomised controlled trials and further real-world studies on different progesterone forms and their combinations are needed. 


\section{CONCLUSION}

The results of our study showed that in FET cycles, vaginal and subcutaneous progesterone provides better pregnancy outcomes than oral progesterone for LPS. Therefore, it may be recommended to use subcutaneous and vaginal forms for LPS in FET cycles.

\section{ETHICAL DECLARATIONS}

Ethics Committee Approval: The study was carried out with the permission of Research Ethics Committee of Erciyes University (number 2020/341, date 24.06.2020).

Informed Consent: Because the study was designed retrospectively, no written informed consent form was obtained from patients.

Referee Evaluation Process: Externally peer-reviewed.

Conflict of Interest Statement: The authors have no conflicts of interest to declare.

Financial Disclosure: The authors declared that this study had received no financial support.

Author Contributions: All of the authors declare that they have all participated in the design, execution, and analysis of the paper and approved the final version.

\section{REFERENCES}

1. Propst AM, Hill JA, Ginsburg ES, Hurwitz S, Politch J, Yanushpolsky EH. A randomized study comparing Crinone 8\% and intramuscular progesterone supplementation in in vitro fertilization-embryo transfer cycles. Fertility and steril 2001; 76: 1144-9.

2. Patki A, Pawar VC. Modulating fertility outcome in assisted reproductive technologies by the use of dydrogesterone. Gynecological Endocrin 2007; 23: 68-72.

3. Nawroth F, Ludwig M. What is the 'ideal'duration of progesterone supplementation before the transfer of cryopreserved-thawed embryos in estrogen/progesterone replacement protocols? Human Reprod 2005; 20: 1127-34.

4. Ludwig M, Diedrich K. Evaluation of an optimal luteal phase support protocol in IVF. Acta Obstetricia Gynecologica Scandina 2001; 80: 452-66.

5. Lee VCY, Li RHW, Ng EHY, Yeung WSB, Ho PC. Luteal phase support does not improve the clinical pregnancy rate of natural cycle frozen-thawed embryo transfer: a retrospective analysis. Eur J Obstet Gynecol Reprod Biol 2013; 169: 50-3.

6. Lan VT, Tuan PH, Canh LT, Tuong HM, Howles CM. Progesterone supplementation during cryopreserved embryo transfer cycles: efficacy and convenience of two vaginal formulations. Reprod Biomed Online 2008; 17: 318-23.

7. Kyrou D, Fatemi HM, Popovic-Todorovic B, Van den Abbeel E, Camus M, Devroey P. Vaginal progesterone supplementation has no effect on ongoing pregnancy rate in hCG-induced natural frozen-thawed embryo transfer cycles. Eur J Obstet Gynecol Reprod Biol 2010; 150: 175-9.

8. Jing S, Li XF, Zhang S, Gong F, Lu G, Lin G. Increased pregnancy complications following frozen-thawed embryo transfer during an artificial cycle. J Assist Reprod Genet 2019; 36: 925-33.
9. Ho $\mathrm{CH}$, Chen SU, Peng FS, Chang CY, Yang YS. Luteal support for IVF/ICSI cycles with Crinone $8 \%(90 \mathrm{mg})$ twice daily results in higher pregnancy rates than with intramuscular progesterone. J Chinese Medical Assoc 2008; 71: 386-91.

10. Vaisbuch E, Leong M, Shoham Z. Progesterone support in IVF: is evidence-based medicine translated to clinical practice? A worldwide web-based survey. Reprod Biomed Online 2012; 25: 139-45.

11. Tomás C, Alsbjerg B, Martikainen H, Humaidan P. Pregnancy loss after frozen-embryo transfer-a comparison of three protocols. Fertil Steril 2012; 98: 1165-9.

12. Salehpour S, Tamimi M, Saharkhiz N. Comparison of oral dydrogesterone with suppository vaginal progesterone for lutealphase support in in vitro fertilization (IVF): A randomized clinical trial. Iranian J Reprod Med 2013; 11: 913.

13. Child T, Leonard SA, Evans JS, Lass A. Systematic review of the clinical efficacy of vaginal progesterone for luteal phase support in assisted reproductive technology cycles. Reprod. Biomed. Online 2018; 36: 630-45.

14. Sator M, Radicioni M, Cometti B, et al. Pharmacokinetics and safety profile of a novel progesterone aqueous formulation administered by the s.c. route. Gynecol Endocrinol 2013; 29: 2058 .

15. Griesinger G, Blockeel C, Tournaye H. Oral dydrogesterone for luteal phase support in fresh in vitro fertilization cycles: a new standard? Fertil Steril 2018 May; 109: 756-62.

16.Zarei A, Sohail P, Parsanezhad ME, Alborzi S, Samsami A, Azizi M. Comparison of four protocols for luteal phase support in frozen-thawed Embryo transfer cycles: a randomized clinical trial. Arch Gynecol Obstet 2017; 295:239-46.

17. Guo W, Chen X, Ye D, et al. Effects of oral dydrogesterone on clinical outcomes of frozen-thawed embryo transfer cycles. Nan fang yi ke da xue xue bao=J Southern Medical Univ 2013; 33: 8615.

18. Rashidi BH, Ghazizadeh M, Tehrani Nejad ES, Bagheri M, Gorginzadeh M. Oral dydrogesterone for luteal support in frozenthawed embryo transfer artificial cycles: a pilot randomized controlled trial. Asian Pac J Reprod 2016; 5: 490-4.

19.Devine K, Richter KS, Widra EA, McKeeby JL. Vitrified blastocyst transfer cycles with the use of only vaginal progesterone replacement with Endometrin have inferior ongoing pregnancy rates: results from the planned interim analysis of a three-arm randomized controlled noninferiority trial. Fertil Steril 2018; 109: 266-75. 